\title{
Two cases of 17a-hydroxylase/17,20-lyase deficiency caused by the CYP17A1 mutation
}

\author{
Hae In Lee, \\ Ahreum Kwon, \\ Jung Hwan Suh, \\ Han Saem Choi, \\ Kyung Chul Song, \\ Hyun Wook Chae, \\ Ho-Seong Kim
}

Department of Pediatrics, Severance Children's Hospital, Endocrine Research Institute, Yonsei University College of Medicine, Seoul, Korea
Received: 12 August, 2020

Revised: 6 September, 2020

Accepted: 28 September, 2020

Address for correspondence:

Ho-Seong Kim

Department of Pediatrics, Severance Children's Hospital, Endocrine Research Institute, Yonsei University College of Medicine, 501 Yonseiro, Seodaemungu, Seoul 03722, Korea Email:kimho@yuhs.ac

https://orcid.org/0000-0003-1135099X 17a-hydroxylase/17,20-lyase deficiency, caused by mutations in the cytochrome P450 family 17 subfamily A member 1 gene (CYP17A1), is an extremely rare form of congenital adrenal hyperplasia that is characterized by diverse phenotypes resulting from specific mutations. Here, we report 2 phenotypic females with 17a-hydroxylase/17,20-lyase deficiency: one with the 46,XX karyotype presenting primary amenorrhea and sexual infantilism, and the other with the $46, X Y$ karyotype presenting a disorder of sexual development. In both cases, the serum levels of adrenocorticotropic hormone, 11-deoxycorticosterone, and gonadotropin were elevated, whereas the levels of testosterone and dehydroepiandrosterone were reduced. Next-generation sequencing revealed one patient with compound heterozygosity for p.Trp17Ter (c.51G >A) and p.His373Leu (c.1118A $>$ T), and the other with homozygosity for p.His373Leu (c.1118A>T). This report further describes 2 cases of 17a-hydroxylase/17,20-lyase deficiency in patients who harbored a p.His373Leu substitution, commonly found in Korean individuals, and presented diverse phenotypes.

Keywords: Congenital adrenal hyperplasia, Steroid 17-a-hydroxylase, Mutation, High-throughput nucleotide sequencing

\section{Highlights}

We introduce 2 cases of patients with 17a-hydroxylase/17,20-lyase deficiency. Both of them harbored a p.His373Leu substitution, providing an evidence of the founder effect of the p.His373Leu substitution in Korean patients with a 17a-hydroxylase/17,20-lyase deficiency.

\section{Introduction}

Congenital adrenal hyperplasia (CAH) is a group of inherited disorders caused by defects in enzymes involved in adrenal steroidogenesis, thereby resulting in various alterations in glucocorticoid, mineralocorticoid, and sex steroid production. ${ }^{1)}$ Among several genes causing $\mathrm{CAH}$, cytochrome P450 family 17 subfamily A member 1 gene (CYP17A1) mutations lead to $17 \alpha$-hydroxylase/17,20-lyase deficiency, which is an extremely rare form of CAH with an estimated worldwide incidence of 1 in 50,000 to 100,000 individuals. ${ }^{2)} 17$-hydroxylase/17,20lyase are involved in androgen and cortisol synthesis from cholesterol; hence, their deficiencies cause androgen and cortisol deficiency, respectively. The diagnosis of a 17a-hydroxylase/17,20lyase deficiency is quite challenging. This rare disorder differs from other forms of $\mathrm{CAH}$, especially from 21-hydroxylase deficiency which accounts for approximately 95\% of CAH cases and is characterized by an unelevated level of 17-hydroxyprogesterone (17-OHprogesterone). Furthermore, patients with $17 a$-hydroxylase/17,20-lyase deficiency usually have mild symptoms and signs of hypocortisolism, and they present primary amenorrhea and/or absence of secondary sexual characteristics during puberty. Furthermore, phenotypes 
largely depend on whether these enzymes are completely or partially deficient. Impaired androgen production results in different phenotypes based on genetic sex: women with the 46,XX karyotype have normal female genitalia but diminished or obliterated sexual development at puberty, while those with the 46,XY karyotype have ambiguous or female external genitalia but no female internal genitalia. Therefore, patients present completely different clinical characteristics based on their karyotypes. Hence, various clinical symptoms should be meticulously assessed and thoroughly considered, and if doubtful, the diagnosis should be confirmed through genetic testing.

Here, we report 2 cases of $17 a$-hydroxylase/17,20-lyase deficiency in women, 1 with the 46,XX karyotype and 1 with the 46,XY karyotype, who harbored CYP17A1 mutations.

\section{Case reports}

\section{Case 1}

A 16-year-old female was transferred to the Department of Pediatric Endocrinology of Severance Children's Hospital for assessment of primary amenorrhea and features of sexual infantilism. The patient had no past medical or family history associated with disorders of sexual development. She was born at a gestational age of 41 weeks with a birth weight of $2.5 \mathrm{~kg}$, compatible with small for gestational age. She was delivered through caesarean section and displayed no typical genital abnormalities postpartum. Initial endocrinological assessment, chromosomal analysis, and imaging investigation were carried out at another hospital. Initial endocrinological investigations revealed a hypergonadotropic hypogonadism with low estradiol and elevated follicle stimulating hormone (FSH) and luteinizing hormone (LH) levels. Decreased cortisol (1.1 $\mu \mathrm{g} /$
dL) and elevated adrenocorticotropic hormone (ACTH) (181.8 $\mathrm{pg} / \mathrm{mL}$ ) levels were noted. The patient's karyotype was 46, XX, and pelvic ultrasonography and pelvic magnetic resonance imaging revealed a small uterus measuring $2 \mathrm{~cm}$ without visible endometrial tissue, a normal vagina, and ovaries with multiple cystic lesions.

She was referred to our endocrinology clinic for further evaluation of primary amenorrhea with primary gonadal failure and suspected hypocortisolism. Physical examination revealed a normal height and weight of $158.3 \mathrm{~cm}$ (25th-50th percentile) and $45.3 \mathrm{~kg}$ (5th-10th percentile), respectively. Her breasts were at Tanner stage II, and pubic hair was at Tanner stage I. No skin pigmentation was observed. Her blood pressure was in the upper normal limit at 129/99 mm Hg (90th-95th percentile). Laboratory investigations revealed normal electrolytes and thyroid function, hypocortisolism (cortisol, $1.9 \mu \mathrm{g} / \mathrm{dL}$; ACTH, $1,815 \mathrm{pg} / \mathrm{mL}$ ), and hypergonadotropic hypogonadism (estradiol, $<8 \mathrm{pg} / \mathrm{ml}$; LH, $47.2 \mathrm{mIU} / \mathrm{mL}$; FSH, $18.2 \mathrm{mIU} / \mathrm{mL}$ ). Meanwhile, she presented a normal $17-\mathrm{OH}$-progesterone $(0.91 \mathrm{ng} / \mathrm{mL})$ and elevated 11-deoxycorticosterone level $(12.30 \mathrm{ng} / \mathrm{mL}$; reference range, $0.02-0.19 \mathrm{ng} / \mathrm{mL}$ ). The aldosterone-to-renin ratio was 39.4 (cutoff, <23.6) with elevated aldosterone levels $(209 \mathrm{pg} / \mathrm{mL}$; reference range, $10-160 \mathrm{pg} / \mathrm{mL}$ ) and low plasma renin activity (PRA) $(0.53 \mathrm{ng} / \mathrm{mL} / \mathrm{hr}$; reference range, $1.31-3.95 \mathrm{ng} / \mathrm{mL} / \mathrm{hr}$ ), indicating hyperaldosteronism (Table 1). After the patient was stimulated with synthetic ACTH, the levels of cortisol, 17-OHprogesterone, 17-hydroxypregnenolone (17-OH-pregnenolone) and dehydroepiandrosterone (DHEA) remained unchanged (Table 1). Based on these results, a 17a-hydroxylase deficiency was suspected, and genetic testing was performed for CYP17A1. Clinical exome sequencing was performed using the TruSight One Sequencing Panel (Illumina, Inc., San Diego, CA, USA), which enriches a $12-\mathrm{Mb}$ region spanning 62,000 target exons of a total 4,813 genes. Compound heterozygous mutations for

Table 1. Hormonal findings at baseline and the results of the synthetic ACTH stimulation test in cases 1 and 2

\begin{tabular}{|c|c|c|c|c|c|}
\hline Variable & \multicolumn{2}{|c|}{ Case $1(46, X X)$} & \multicolumn{2}{|c|}{ Case $2(46, X Y)$} & Reference range \\
\hline \multicolumn{6}{|l|}{ At baseline } \\
\hline 11-Deoxycorticosterone (ng/mL) & \multicolumn{2}{|c|}{12.30} & \multicolumn{2}{|c|}{0.46} & $0.02-0.19$ \\
\hline Aldosterone $(\mathrm{pg} / \mathrm{mL})$ & \multicolumn{2}{|c|}{209} & \multicolumn{2}{|c|}{140} & $10-160$ \\
\hline PRA (ng/mL/hr) & \multicolumn{2}{|c|}{0.53} & \multicolumn{2}{|c|}{0.10} & $1.31-3.95$ \\
\hline Cortisol ( $\mu \mathrm{g} / \mathrm{dL})$ & \multicolumn{2}{|c|}{1.9} & \multicolumn{2}{|c|}{0.7} & $2.8-16.4$ \\
\hline ACTH $(p g / m L)$ & \multicolumn{2}{|c|}{$1,815.0$} & \multicolumn{2}{|c|}{112.0} & $7.2-63.3$ \\
\hline Testosterone (ng/dL) & \multicolumn{2}{|c|}{$<2.5$} & \multicolumn{2}{|c|}{$<2.5$} & $8.4-48.1$ \\
\hline Estradiol (pg/mL) & \multicolumn{2}{|c|}{$<8$} & \multicolumn{2}{|c|}{$<8$} & $50-482$ \\
\hline $\mathrm{LH}(\mathrm{mlU} / \mathrm{mL})$ & \multicolumn{2}{|c|}{47.2} & \multicolumn{2}{|c|}{28.6} & $1.9-12.5$ \\
\hline $\mathrm{FSH}(\mathrm{mlU} / \mathrm{mL})$ & \multicolumn{2}{|c|}{18.2} & \multicolumn{2}{|c|}{70.8} & $2.5-10.2$ \\
\hline ACTH stimulation test & Before & After & Before & After & \\
\hline Progesterone (ng/mL) & - & - & 5.97 & 9.97 & $0.2-0.5$ \\
\hline 17a-OH-pregnenolone (ng/mL) & 0.82 & 1.03 & 1.79 & 2.15 & $0.3-4.4$ \\
\hline 17-OH-progesterone (ng/mL) & 0.91 & 0.98 & 1.09 & 1.17 & $0.2-1.0$ \\
\hline Cortisol $(\mu \mathrm{g} / \mathrm{dL})$ & 1.7 & 1.8 & 0.7 & 1.5 & $2.8-16.4$ \\
\hline DHEA (ng/mL) & $<0.50$ & $<0.50$ & $<0.1$ & $<0.1$ & $1.3-9.8$ \\
\hline
\end{tabular}

ACTH, adrenocorticotrophic hormone; PRA, plasma renin activity; LH, luteinizing hormone; FSH, follicle stimulating hormone; DHEA, dehydroepiandrosterone. 
p.Trp17Ter $(\mathrm{c} .51 \mathrm{G}>\mathrm{A})$ and p.His373Leu $(\mathrm{c} .1118 \mathrm{~A}>\mathrm{T})$ were identified in CYP17A1 (Fig. 1A). As both mutations were evaluated as pathogenic according to the 2015 ACMG-AMP guidelines, ${ }^{4)}$ the diagnosis of a $17 \alpha$-hydroxylase/17,20-lyase deficiency was confirmed.

After receiving the diagnosis of a 17a-hydroxylase/17,20-lyase deficiency, the patient started oral hydrocortisone treatment $(10$ $\mathrm{mg} / \mathrm{m}^{2} /$ day). Furthermore, Femoston, a combination of 2-mg estradiol and 10-mg progesterone, was initiated. Four months after treatment initiation, physical examination revealed Tanner stage III breasts and Tanner stage II pubic hair, her blood pressure decreased to $110 / 70 \mathrm{~mm} \mathrm{Hg}$ (25th-50th percentile), and she began menstruation. Laboratory tests revealed normal levels of cortisol $(6.9 \mu \mathrm{g} / \mathrm{dL})$, ACTH $(5.9 \mathrm{pg} / \mathrm{mL})$, estradiol $(90.84 \mathrm{pg} / \mathrm{mL}), \mathrm{LH}(6.16 \mathrm{mIU} / \mathrm{mL})$, and FSH $(8.3 \mathrm{mIU} / \mathrm{mL})$ at a 6-month follow-up evaluation. Furthermore, transabdominal sonography 6 months after treatment revealed a normal uterus measuring $15.9 \mathrm{~mL}$ (normal range, $21.2 \pm 13.5 \mathrm{~mL}$ ).

\section{Case 2}

A 4-year-old phenotypic female patient who presented with a bulging right inguinal area underwent right hernioplasty. As the hernia sac displayed prepubertal testis without ovarian stroma, she was referred to our endocrinology clinic for further evaluation of disorders of sexual development. She was born at full-term through caesarean section, and her birth weight was $3.41 \mathrm{~kg}$. She did not have a specific medical or family history. Physical examination revealed normal female external genitalia with a normal clitoris, urethra, and vaginal opening. Her growth status was normal, with a height and weight of

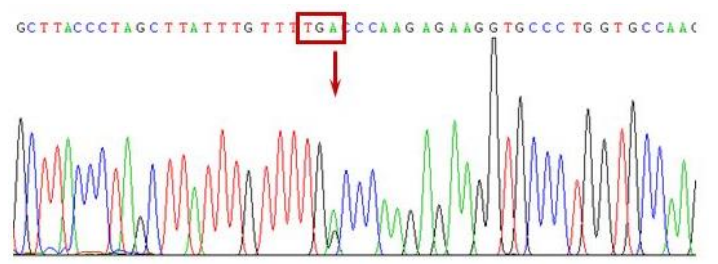

p.Trp17Ter (c.51G>A)

CG TGGCCCCTATGCTCATCCCDCTC AGGCCAACG TTG AC TCCAGGTGTG

(A)
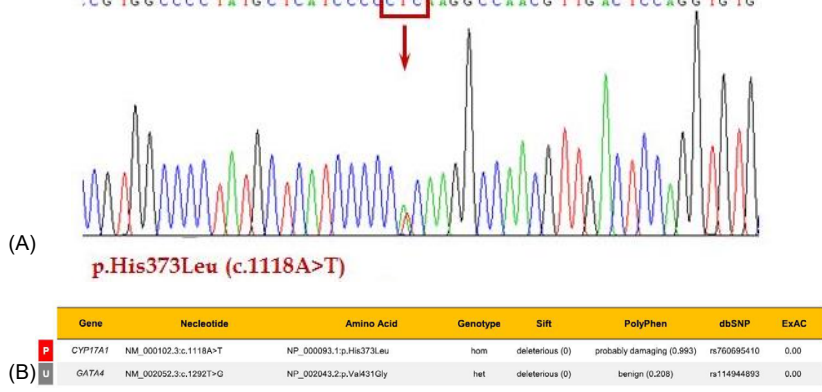

Fig. 1. (A) Direct sequencing of the CYP17A1 gene from the patient in case 1 . Heterozygous p.Trp17Ter (c.51G>A), p.His373Leu (c,1118A>T) mutations were identified. (B) Next-generation sequencing for hypogonadism from the patient in case 2. A homozygous p.His373Leu (c.1119A>T) CYP17A1 mutation was identified. P, pathogenic; U, uncertain significance.
$115 \mathrm{~cm}$ and $23.9 \mathrm{~kg}$ (90th-95th percentile in age-matched girl), respectively. Her blood pressure was 145/100 mm Hg ( $>97$ th percentile). Initial endocrinological investigations were as follows: $\mathrm{LH}, 28.6 \mathrm{mIU} / \mathrm{mL}$; FSH, $70.8 \mathrm{mIU} / \mathrm{mL}$; testosterone, $<2.5 \mathrm{ng} / \mathrm{dL}$; estradiol, $<8 \mathrm{pg} / \mathrm{mL}$; cortisol, $0.7 \mu \mathrm{g} /$ $\mathrm{dL}$ (reference range, $2.8-16.4 \mu \mathrm{g} / \mathrm{dL}$ ); ACTH, $112.0 \mathrm{pg} / \mathrm{mL}$ (reference range, $7.2-63.3 \mathrm{pg} / \mathrm{mL}$ ); serum aldosterone, $140 \mathrm{pg} /$ $\mathrm{mL}$ (reference range, $10-160 \mathrm{pg} / \mathrm{mL}$ ); and PRA, $0.10 \mathrm{ng} / \mathrm{mL} / \mathrm{hr}$ (reference range, $1.31-3.95 \mathrm{ng} / \mathrm{mL} / \mathrm{hr}$ ). The findings indicated mild hypocortisolism and elevated gonadotropin (Table 1). Meanwhile, she presented a normal 17-OH-progesterone (1.09 $\mathrm{ng} / \mathrm{mL}$ ) and elevated progesterone $(5.97 \mathrm{ng} / \mathrm{mL}$; reference range, $0.2-0.5 \mathrm{ng} / \mathrm{mL})$ and 11 -deoxycorticosterone level $(0.46$ $\mathrm{ng} / \mathrm{mL}$; reference range, $0.02-0.19 \mathrm{ng} / \mathrm{mL}$ ). The aldosteroneto-renin ratio was 140 , indicating hyperaldosteronism, although an electrolyte imbalance was not observed. A highdose synthetic ACTH stimulation test revealed no response of cortisol, 17-OH-progesterone, 17-OH-pregnenolone, and DHEA, but rather revealed an elevated progesterone response (from 5.97 to $9.97 \mathrm{ng} / \mathrm{mL}$ ) (Table 1). Chromosomal analysis and fluorescence in situ hybridization revealed a normal male karyotype, 46,XY, with SRY. Pelvic ultrasonography revealed no visible uterus or ovaries in the pelvic cavity, and genitography revealed a normal urethral and vaginal opening. With the suspicion of 17a-hydroxylase/17,20-lyase deficiency, we performed next-generation sequencing-based targeted gene panel for hypogonadism that encompassed 36 related genes (AKAP6, AMH, CDKL5, CGA, CRK, CYP17A1, CYP19A1, CYP1A1, DLK1, ESR1, FSHR, GH1, GHRH, GNAS, HRAS, IGF1, IGFALS, INS, KISS1, KISS1R, LEP, LEPQTL1, LEPR, LHB, LHCGR, LIN28B, MKRN3, NF1, NFIX, NNT, NOTCH1, NROB1, OGDHL, PROKR2, PTPN11, and STK11) using Miseq (Illumina, Inc.). A homozygous p.His373Leu (c.1118A $>$ T) mutation of the CYP17Al gene was identified, confirming 17a-hydroxylase/17,20-lyase deficiency (Fig. 1B).

Oral hydrocortisone $\left(12 \mathrm{mg} / \mathrm{m}^{2} /\right.$ day $)$ treatment was initiated. Given her clinical features, sex of rearing, and parents' opinion, the patient was raised as a girl, and she underwent orchiectomy on the opposite side. Pathological assessment revealed a prepubertal testis with no evidence of residual germ cells and slightly increased Leydig cells. After treatment with hydrocortisone, the patient's blood pressure and serum levels of cortisol and ACTH remained within normal ranges. Lowdose oral estradiol treatment was initiated at 12 years of age to promote the development of secondary sexual characteristics.

\section{Discussion}

17a-hydroxylase/17,20-lyase deficiency is a rare form of $\mathrm{CAH}$, accounting for only approximately $1 \%$ of all $\mathrm{CAH}$ cases. ${ }^{2,5}$ The first reported case was a 35 -year-old patient in San Francisco in 1966 who was both genotypically and phenotypically female but presented with sexual infantilism and primary amenorrhea. ${ }^{6}$ As patients with this disorder can present various phenotypes based on the types of mutations, genetic 
analysis is necessary. Hence, a thorough pathophysiological understanding of the clinical manifestations and molecular derangements is required. CYP17A1 (10q24.3) encodes the P450c17 enzyme, a type 2 microsomal P450 enzyme expressed in the adrenal gland and gonads, which performs both steroid 17 -hydroxylase and 17,20-lyase activities. ${ }^{5,7)}$ Both enzymes contribute to cortisol and sex steroid production through the steroid biosynthesis pathway. 17a-hydroxylase converts pregnenolone to 17-OH-pregnenolone and progesterone to 17-OH-progesterone, and 17,20-lyase further converts to DHEA and androstenedione. Defects in 17 $\alpha$-hydroxylase/17,20lyase result in a reduction in the 17-OH-pregnenolone and 17-OH-progesterone levels, resulting in low cortisol levels and a decline in DHEA and androstenedione, which in turn result in low adrenal androgen levels. Low cortisol levels result in the overstimulation of the steroid synthetic pathway as negative feedback, resulting in ACTH overproduction and adrenal cortex hyperplasia. Since CYP17A1 is expressed in the zona reticularis and zona fasciculata of the adrenal gland, but not in the zona glomerulosa, steroidogenesis through ACTH stimulation leads to deoxycorticosterone and corticosterone overproduction. ${ }^{3)}$ Elevated deoxycorticosterone levels lead to sodium retention, hypokalemia, hypertension, and suppression of aldosterone production. Although cortisol production is low or absent in 17a-hydroxylase/17,20-lyase deficiency, adrenal disorders seldom occur owing to the presence of corticosterone, which has glucocorticoid activity.

As of September 2020, 123 CYP17A1 mutations have been identified in the Human Gene Mutation Database. The first mutation was reported in a Thai patient of Chinese descent as a 4-base insertion in 1993. ${ }^{8)}$ Different populations harbor different mutations with regional accumulation as a founder effect. Since the first report of a homozygous missense p.His373Leu substitution in 2 Japanese sisters in $1993,{ }^{9)}$ the p.His373Leu mutation has been frequently reported in Japanese, Chinese, and Korean individuals, which is presumed to exert a founder effect in Northeast Asian populations. ${ }^{10,11)}$ Since the first $p$.His373Leu mutation in a Korean individual was reported in 2012, 10 cases, including the present 2 patients, have been reported to carry the CYP17A1 mutations with the p.His373Leu substitution in the Korean population. ${ }^{10,12,13)}$ These Korean cases provide robust evidence that the p.His373Leu substitution exerts a founder effect, especially in Korean patients with a 17a-hydroxylase/17,20-lyase deficiency. The previous functional study revealed that COS-1 cells transfected with a p.His373Leu mutant obliterate both $17 \alpha$-hydroxylase and 17,20-lyase activities. ${ }^{9)}$ It has been proposed that p.His373Leu substitution does not allow for appropriate incorporation of the heme moiety, leading to a global alteration of P450c17 structure and consequently prevents heme binding, thus attenuating enzyme activity. ${ }^{9,14)}$ Furthermore, a previous study reported that the p.His373Leu mutant did not have 11ß-hydroxylase and aldosterone synthase activities since the mutant failed to convert $\left[{ }^{14} \mathrm{C}\right]$ deoxycorticosterone to corticosterone or aldosterone. ${ }^{9)}$ Most cases of 17a-hydroxylase/17,20-lyase deficiency revealed low aldosterone levels owing to suppression of the renin-angiotensin system. However, our patients displayed discrepancies, revealing high aldosterone levels with suppressed PRA, similar with previous reports. ${ }^{9,12)}$ While the reason underlying this difference remains unclear, it is speculated that the presence of low-level enzyme activity based on the genotype or unknown factors contribute to phenotypic variation. ${ }^{15)}$ Furthermore, Monno et al. reported that aldosterone secretion may be influenced by an increase in ACTH levels and not by the activation of the renin-angiotensin system. ${ }^{9}$ Like glucocorticoid-remediable aldosteronism, which has a chimeric $11 \beta$-hydroxylase/aldosterone synthase carrying out aldosterone synthesis with cortisol and cortisol precursors ${ }^{16)}$ plasma aldosterone levels in patients with 17a-hydroxylase/17,20-lyase deficiency appear to be regulated by ACTH.

In conclusion, this case report describes 2 additional cases of CYP17A1 mutations in Korea, both harboring a p.His373Leu substitution. One harbored a heterozygous mutation, and the other harbored a homozygous mutation. It may be speculated that the founder effect of the p.His373Leu substitution manifests among Korean individuals harboring CYP17A1 mutations. Because patients with $17 a$-hydroxylase/17,20-lyase deficiency present variable clinical characteristics based on their karyotypes, meticulous assessment including genetic testing is necessary.

\section{Ethical statement}

This case was approved by the Institutional Review Board of Yonsei University Health System, Severance Hospital (approval number: 4-2020-0604). Informed consents were obtained from the patients and their parents.

\section{Conflict of interest}

No potential conflict of interest relevant to this article was reported.

\section{References}

1. Turcu AF, Auchus RJ. Adrenal steroidogenesis and congenital adrenal hyperplasia. Endocrinol Metab Clin North Am 2015;44:275-96.

2. Oh YK, Ryoo U, Kim D, Cho SY, Jin DK, Yoon BK, et al. 17alpha-hydroxlyase/17, 20-lyase deficiency in three siblings with primary amenorrhea and absence of secondary sexual development. J Pediatr Adolesc Gynecol 2012;25:e103-5.

3. El-Mauche D AW, Merke DP. Congenital adrenal hyperplasia. Lancet 2017;390:2194-210.

4. Richards S, Aziz N, Bale S, Bick D, Das S, Gastier-Foster $\mathrm{J}$, et al. Standards and guidelines for the interpretation of sequence variants: a joint consensus recommendation of the American College of Medical Genetics and Genomics 
and the Association for Molecular Pathology. Genet Med 2015;17:405-24.

5. Miller WL. Mechanisms in endocrinology: rare defects in adrenal steroidogenesis. Eur J Endocrinol 2018;179:R12541.

6. Biglieri EG, Herron MA, Brust N. 17-hydroxylation deficiency in man. J Clin Invest 1966;45:1946-54.

7. Xu S, Hu S, Yu X, Zhang M, Yang Y. 17alphahydroxylase/ 17,20lyase deficiency in congenital adrenal hyperplasia: a case report. Mol Med Rep 2017;15:339-44.

8. Fardella CE, Zhang LH, Mahachoklertwattana P, Lin D, Miller WL. Deletion of amino acids Asp487-Ser488Phe489 in human cytochrome P450c17 causes severe 17 alpha-hydroxylase deficiency. J Clin Endocrinol Metab 1993;77:489-93.

9. Monno S, Ogawa H, Date T, Fujioka M, Miller WL, Kobayashi M. Mutation of histidine 373 to leucine in cytochrome P450c17 causes 17 alpha-hydroxylase deficiency.J Biol Chem 1993;268:25811-7.

10. Kim YM, Kang M, Choi JH, Lee BH, Kim GH, Ohn JH, et al. A review of the literature on common CYP17A1 mutations in adults with 17-hydroxylase/17,20-lyase deficiency, a case series of such mutations among Koreans and functional characteristics of a novel mutation. Metabolism 2014;63:429.
11. Qiao J, Hu RM, Peng YD, Song HD, Peng YW, Gao GF, et al. A complex heterozygous mutation of His373Leu and Asp487-Ser488-Phe489 deletion in human cytochrome P450c17 causes 17alpha-hydroxylase/17,20-lyase deficiency in three Chinese sisters. Mol Cell Endocrinol 2003;201:189-95.

12. Lee MH, Won Park S, Yoon TK, Shim SH. Homozygous CYP17A1 mutation (H373L) identified in a 46,XX female with combined 17a-hydroxylase/17,20-lyase deficiency. Gynecol Endocrinol 2012;28:573-6.

13. Kim SM, Rhee JH. A case of 17 alpha-hydroxylase deficiency. Clin Exp Reprod Med 2015;42:72-6.

14. Auchus RJ, Miller WL. Molecular modeling of human P450c17 (17alpha-hydroxylase/17,20-lyase): insights into reaction mechanisms and effects of mutations. Mol Endocrinol 1999;13:1169-82.

15. Costa-Santos M, Kater CE, Auchus RJ; Brazilian Congenital Adrenal Hyperplasia Multicenter Study Group. Two prevalent CYP17 mutations and genotype-phenotype correlations in 24 Brazilian patients with 17-hydroxylase deficiency. J Clin Endocrinol Metab 2004;89:49-60.

16. Lifton RP, Dluhy RG, Powers M, Rich GM, Cook S, Ulick S, et al. A chimaeric 11 beta-hydroxylase/aldosterone synthase gene causes glucocorticoid-remediable aldosteronism and human hypertension. Nature 1992;355:262-5. 